\title{
Cancer gene therapies come of age
}

\author{
"Improving efficiency of transgene expression and vector spread, and, at the same time, making the vectors safer will allow the \\ development of approved medicines. Gene therapy in combination with other treatment modalities ... will further boost the \\ success rate of such treatments."
}

In contrast to many other diseases, 'cancer' comprises a whole group of quite heterogenous malignancies, differing in their genetic background, biochemistry and physiology. Even within one individual, metastases or reccurring tumors differ from the primary lesion, making it almost impossible to find a generalized cure for this disease. Nevertheless, there are several common features in tumors defined as hallmarks of the disease, which could offer novel and more generalized points of intervention [1]. Classical treatment regimes, such as surgical resection (in the case of solid tumors), followed by radiation and/or chemotherapy have, for the most part, worked out well for primary lesions, whereas in cases of metastasized disease and relapse, these treatment regimes often fail due to the occurrence of resistance mechanisms. In these situations, novel therapies, for example those based on nucleic acids, can offer promising treatment options. Nowadays, with more and more clinical trials having a successful outcome, public acceptance of cancer gene therapy is increasing and nonprofit organizations such as the Alliance for Cancer Gene Therapy [101] have been founded to promote gene therapy research for cancer.

In cancer gene therapy, the nucleic acid acts as an inactive prodrug that can be converted to the active drug; for example, the therapeutically active protein at the tumor site. Selectivity for tumor tissue and tumor cells can be achieved by several means. Delivery of nucleic acids into tumors can be simply accomplished by topical application into or close to the tumor. This is achievable in the case of localized disease, whereas for tumors that are already disseminated or inaccessible, the intravenous route is inevitable. Systemic targeting of therapeutic nucleic acid to tumors can be achieved by using suitable carrier systems. To prevent their rapid elimination from the bloodstream after intravenous injection, such systems can be rendered more or less 'stealth'-like allowing their circulation in blood and their accumulation in the tumor tissue, which often possess a leaky vasculature [2]. Further on, targeting of these carriers to tumor cell-surface markers will enable a high level of selectivity at the cellular level. For example, gene vectors carrying growth factor receptor binding ligands are selectively bound to and internalized by tumor cells that over-express growth factor receptor $[3,4]$. Once inside the cells, transcription of the transgene into the actual therapeutically active protein allows an additional level of selectivity. Several promoters, which are usually upregulated during embryogenesis, are re-activated in malignant cells and can be harnessed to specifically control transgene expression in tumor cells $[5,6]$.

A multitude of gene vectors have been developed for efficiently transporting nucleic acids into tumor cells, and also to the tumor site using systemic application for metastasized disease. In principle, two major types of carriers can be defined, namely recombinant viruses and synthetic gene delivery systems. In terms of cancer gene therapy, both gene delivery systems have evolved over the last 20 years of research, so that several nonviral and viral vectors are now in advanced clinical studies with some of them close to approval medicines. Since the first clinical trials in the early 1990s, more than 1000 clinical trials have been conducted in the field of cancer gene therapy [102]. Besides ex vivo transduction of cells (e.g., tumor-infiltrating lymphocytes), direct intratumoral application of the gene vector was conducted. In the virus vector field, early clinical studies were carried out with replication-incompetent retroviruses [7] or adenoviruses [8] carrying a therapeutic gene under the control of a strong, ubiquitous promoter. Owing to the lack of control of transgene expression, ubiquitous promoters such as the cytomegalovirus promoter are prone to rapid inactivation in vivo due to promoter

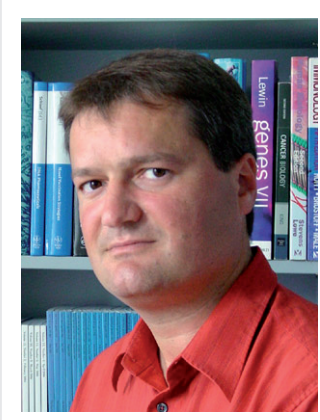

\section{Manfred Ogris}

Department of Pharmacy, Center for Drug Research, Pharmaceutical Biotechnology, Ludwig-Maximilians University, Butenand str. 5-13, D-81377 Munich, Germany Tel.: +49892 18977842 Fax: +4989218977791 E-mail: manfred.ogris@ cup.uni-muenchen.de 
methylation limiting the duration of transgene expression [9]. Long-term follow-up studies in cancer patients suggest that the positive effect of such first-generation viral vectors is largely due to the immune stimulatory effects of the virus injected, as the expression of the therapeutic transgene was short lived and the level of transfected cells low [10]. To overcome limitations due to limited transgene expression and virus spread within the tumor, viral vectors that can selectively replicate in tumor cells were developed. ONYX 015 is an adenoviral vector selectively replicating in tumors with a defective p53 pathway that cannot replicate in healthy tissue; but other mechanisms are also important for this tumor selectivity [11]. Promising results were obtained in combination with chemotherapeutics in a Phase II clinical trial [12], although the efficacy of the virus alone is rather limited [13]. Several other virus strains are currently being evaluated for cancer-selective replication and killing, including measles, vaccinia, pox and Newcastle virus [14]. In a recent review by Roberto Cattaneo and colleagues, the concept of targeted, armed and shielded viruses for cancer therapy was discussed [15]:

- Modifying the virus' surface with shielding polymers allows changing the wild-type virus' tropism after intravenous injection (e.g., liver for adenovirus), and use of tumor-selective ligands for specific uptake by tumor cells;

- Local activation of viruses can be achieved at the tumor site by tumor-secreted proteases;

- Tumor-selective replication is achieved by tumor-specific promoters;

- Finally, the virus is armed with a therapeutic gene, which, encodes for an enzyme that activates a prodrug into the toxic product at the tumor site.

In the field of nonviral vectors, the major limitation is still their low efficiency of transgene expression in vivo. Several clinical trials have been conducted with 'naked' DNA, solely injecting the plasmid into the tumor tissue with limited success [16]. To improve efficiency, plasmids were packed into nonviral carriers based on liposomes and cationic polymer, where in a Phase I trial on ovary cancer, transgene expression (IL-12) was found in tumor tissue [17]. Tumor-selective expression of highly toxic diphtheria toxin was achieved with a plasmid vector in combination with the polycation polyethylenimine locally applied in bladder cancer [18]. Although only a Phase I trial, the treatment led to tumor ablation in four out of 18 patients treated and to a tumor reduction of at least $50 \%$ in eight patients. As with to viral vectors, tumor specificity can be achieved by shielding, cell targeting and transcriptional targeting. Unfortunately, shielding the vector often further reduces efficiency [19], and the compaction of nucleic acids with cation lipids or polycations has to be designed to be reversible, otherwise the access of the transferred DNA to the intracellular transcription machinery or the release of RNA (e.g., siRNA and micro RNA [miRNA]) into the cytoplasm will be limited [20]. Here, the concept of 'artificial viruses', which sense their environment and become fully active; for example, as at the tumor site, can help in making synthetic gene delivery vectors more efficient without losing specificity [21]. Chemical or enzymatic trigger mechanisms allow intracellular or tumor-environment-specific cleavage of a shielding polymer, the polymer can be cleaved by tumor-specific proteases, such as matrix metalloproteases [22], or lowered endosomal pH inside the cell [19]. Triggered release of nucleic acid inside the cells can be achieved by using carrier molecules containing intracellular cleavage sites, such as disulfides or diesters [23].

\section{Cancer gene therapy approaches in advanced clinical studies (Phase III \& beyond)}

At present, several viral and nonviral gene vectors are undergoing advanced clinical studies. For nonviral vectors, allovectin-7, a liposomaly formulated plasmid encoding for immune stimulatory proteins [24], is the most clinically advanced vector currently being applied in Phase III in combination with chemotherapy [103]. Replication-defective adenovirus expressing the cytotoxic cytokine TNF- $\alpha$ (TNFerade $\left.{ }^{\circledR}\right)$ in now also being evaluated in a Phase III trial and could become an approved medicine for cancer treatment [25]. Gendicine, a recombinant, replicationdefective adenovirus encoding for the tumor suppressor p53 has been approved by Chinese authorities for treatment of several cancer types. In the USA, a biological license application has been submitted for p53-encoding, replicationdefective Adenovirus (Advexin ${ }^{\circledR}$ ) for treatment of head and neck cancer [104].

\section{Future perspective}

Oncolytic vectors that selectively replicate in tumor tissue are a key step in the clinical application of tumor-selective gene carriers, in that they harness genetic differences between healthy 
and malignant tissue, allowing tumor-cell-specific killing (as described previously). A further possibility to reduce off-target activity of vectors used for cancer gene therapy is to use miRNA based technologies. Some years ago miRNA was identified as a key player in post-transcriptional regulation of gene expression [26]. Over 700 different miRNAs are already described in humans and their distribution pattern in different tissues has been analyzed [27]. After binding to its target sequence at the 3'UTR end of the mRNA molecule, a protein complex leads to site-specific cleavage and, hence, inactivation of the mRNA. For example, a recombinant lentivirus containing binding sites for miRNA upregulated in hematopoietic cells prevented transduction of this cell type in vivo, but allowed long lasting transgene expression in the liver [28]. miRNA dysregulation is frequently found to be a cause for the oncogenic potential of malignant tumors[29]. Currently, research is conducted in utilizing infectious wildtype virus (which usually destroys its target cells after infection) made tumor-specific by controlling its replication via miRNA levels being aberrant in tumor tissue. For example, miRNA122 is usually present at high levels in hepatocytes, but is downregulated in hepatocellular carcinoma [30]. When inserting the miRNA122 binding site into the 3'UTR of the E1A region of wild-type adenovirus, hepatic virus replication was considerably decreased, whereas activity in hepatocellular cancer cells in vitro was unaffected [31]. In a similar study with herpes simplex virus, the binding site for the ubiquitously expressed miRNA143 or miRNA145 prevented virus replication in healthy tissue, whereas prostate cancer cells with very low levels of these miRNAs were selectively killed by the virus [32].

\section{Conclusion}

With all the ups and down, within 20 years of research, cancer gene therapy will become an important option for the treatment of malignant disease. Improving efficiency of transgene expression and vector spread, and, at the same time, making the vectors safer will allow the development of approved medicines. Gene therapy in combination with other treatment modalities, such as chemotherapy and/or radiation, will further boost the success rate of such treatments.

\section{Financial \& competing interests disclosure}

The author has no relevant affiliations or financial involvement with any organization or entity with a financial interest in or financial conflict with the subject matter or materials discussed in the manuscript. This includes employment, consultancies, honoraria, stock ownership or options, expert testimony, grants or patents received or pending, or royalties.

No writing assistance was utilized in the production of this manuscript.

\section{Bibliography}

1 Hanahan D, Weinberg RA. The hallmarks of cancer. Cell 100(1), 57-70 (2000).

2 Iyer AK, Khaled G, Fang J, Maeda $H$. Exploiting the enhanced permeability and retention effect for tumor targeting. Drug Discov.Today 11(17-18), 812-818 (2006).

3 De Bruin K, Ruthardt N, Von Gersdorff K et al. Cellular dynamics of EGF receptortargeted synthetic viruses. Mol. Ther. 15(7), 1297-1305 (2007).

4 Morrison J, Briggs SS, Green NK et al. Cetuximab retargeting of adenovirus via the epidermal growth factor receptor for treatment of intraperitoneal ovarian cancer. Hum. Gene Ther. 20(3), 239-251 (2009).

5 Fukazawa T, Matsuoka J, Yamatsuji T, Maeda Y, Durbin ML, Naomoto Y. Adenovirus-mediated cancer gene therapy and virotherapy. Int. J. Mol. Med. 25(1), 3-10 (2010)

6 Dorer DE, Nettelbeck DM. Targeting cancer by transcriptional control in cancer gene therapy and viral oncolysis. Adv. Drug Deliv. Rev. 61(7-8), 554-571 (2009).
7 Ram Z, Culver KW, Oshiro EM et al. Therapy of malignant brain tumors by intratumoral implantation of retroviral vector-producing cells. Nat. Med. 3(12), 1354-1361 (1997).

8 Sterman DH, Treat J, Litzky LA et al. Adenovirus-mediated herpes simplex virus thymidine kinase/ganciclovir gene therapy in patients with localized malignancy: results of a Phase I clinical trial in malignant mesothelioma. Hum. Gene Ther. 9(7), 1083-1092 (1998).

9 Gerolami R, Uch R, Jordier F et al. Gene transfer to hepatocellular carcinoma: transduction efficacy and transgene expression kinetics by using retroviral and lentiviral vectors. Cancer Gene Ther. 7(9), 1286-1292 (2000).

10 Sterman DH, Recio A, Vachani A et al. Long-term follow-up of patients with malignant pleural mesothelioma receiving high-dose adenovirus herpes simplex thymidine kinase/ganciclovir suicide gene therapy. Clin. Cancer Res. 11(20), 74447453 (2005).
11 Dix BR, Edwards SJ, Braithwaite AW. Does the anti-tumor adenovirus onyx-015/d11520 selectively target cells defective in the p53 pathway? J. Virol. 75(12), 5443-5447 (2001).

12 Khuri FR, Nemunaitis J, Ganly I et al. A controlled trial of intratumoral ONYX-015, a selectively-replicating adenovirus, in combination with cisplatin and 5-fluorouracil in patients with recurrent head and neck cancer. Nat. Med. 6(8), 879-885 (2000).

13 Kirn D. Clinical research results with dl1520 (ONYX-015), a replication-selective adenovirus for the treatment of cancer: what have we learned? Gene Ther. 8(2), 89-98 (2001).

14 Crompton AM, Kirn DH. From ONYX-015 to armed vaccinia viruses: the education and evolution of oncolytic virus development. Curr. Cancer Drug Targets 7(2), 133-139 (2007).

15 Cattaneo R, Miest T, Shashkova EV, Barry MA. Reprogrammed viruses as cancer therapeutics: targeted, armed and shielded. Nat. Rev. Microbiol. 6(7), 529-540 (2008). 
16 Heinzerling L, Burg G, Dummer R et al. Intratumoral injection of DNA encoding human interleukin 12 into patients with metastatic melanoma: clinical efficacy. Hum. Gene Ther. 16(1), 35-48 (2005).

17 Anwer K, Barnes MN, Fewell J, Lewis DH, Alvarez RD. Phase I clinical trial of IL-12 plasmid/lipopolymer complexes for the treatment of recurrent ovarian cancer. Gene Ther. 17(3), 360-369 (2010).

18 Sidi AA, Ohana P, Benjamin S et al. Phase I/II marker lesion study of intravesical Bc-819 DNA plasmid in H19 over expressing superficial bladder cancer refractory to bacillus calmette-guerin. J. Urol. 180(6), 2379-2383 (2008)

19 Walker GF, Fella C, Pelisek J et al. Toward synthetic viruses: endosomal $\mathrm{pH}$-triggered deshielding of targeted polyplexes greatly enhances gene transfer in vitro and in vivo. Mol. Ther. 11(3), 418-425 (2005).

20 Chen $\mathrm{HH}$, Ho YP, Jiang X, Mao HQ, Wang TH, Leong KW. Quantitative comparison of intracellular unpacking kinetics of polyplexes by a model constructed from quantum dot-FRET. Mol. Ther. 16(2), 324-332 (2008).

21 Edinger D, Wagner E. Bioresponsive polymers for the delivery of therapeutic nucleic acids. Wiley Interdiscip. Rev. Nanomed. Nanobiotechnol. DOI: 10.1002/wnan.97 (2010) (Epub ahead of print).
22 Hatakeyama H, Akita H, Kogure K et al. Development of a novel systemic gene delivery system for cancer therapy with a tumorspecific cleavable PEG-lipid. Gene Ther. 14(1), 68-77 (2007).

23 Kloeckner J, Wagner E, Ogris M. Degradable gene carriers based on oligomerized polyamines. Eur. J. Pharm. Sci. 29(5), 414-425 (2006)

24 Bedikian AY, Del Vecchio M. Allovectin-7 therapy in metastatic melanoma. Expert Opin. Biol. Ther. 8(6), 839-844 (2008).

25 Weichselbaum RR, Kufe D. Translation of the radio- and chemo-inducible TNFerade vector to the treatment of human cancers. Cancer Gene Ther. 16(8), 609-619 (2009).

26 He L, Hannon GJ. MicroRNAs: small RNAs with a big role in gene regulation. Nat. Rev Genet. 5(7), 522-531 (2004).

27 Landgraf P, Rusu M, Sheridan R et al. A mammalian microRNA expression atlas based on small RNA library sequencing. Cell 129(7), 1401-1414 (2007).

28 Brown BD, Cantore A, Annoni A et al. A microRNA-regulated lentiviral vector mediates stable correction of hemophilia B mice. Blood 110(13), 4144-4152 (2007).

29 Croce CM. Causes and consequences of microRNA dysregulation in cancer. Nat. Rev. Genet. 10(10), 704-714 (2009).
30 Coulouarn C, Factor VM, Andersen JB, Durkin ME, Thorgeirsson SS. Loss of miR-122 expression in liver cancer correlates with suppression of the hepatic phenotype and gain of metastatic properties. Oncogene 28(40), 3526-3536 (2009).

31 Cawood R, Chen HH, Carroll F, Bazan-Peregrino M, Van Rooijen N, Seymour LW. Use of tissue-specific microRNA to control pathology of wild-type adenovirus without attenuation of its ability to kill cancer cells. PLoS Pathog. 5(5), e1000440 (2009).

32 Lee CY, Rennie PS, Jia WW. MicroRNA regulation of oncolytic herpes simplex virus-1 for selective killing of prostate cancer cells. Clin. Cancer Res. 15(16), 5126-5135 (2009).

\section{- Websites}

101 Alliance for Cancer Gene Therapy http://www.acgtfoundation.org/

102 Gene therapy clinical trials worldwide www.wiley.co.uk/genetherapy/clinical/

103 Clinicaltrial.gov. Trial number NCT00395070 http://www.clinicaltrials.gov/ct2/show/ NCT00395070

104 Nature reviews drug discovery news and analysis http://www.nature.com/nrd/journal/v7/n8/ full/nrd2650.html\#First-adenovirusgenetherapy-application-submitted-to-FDA 